\title{
Overview of the socio-economic consequences of heart failure
}

\author{
Franz P. Hessel \\ SRH Berlin University of Applied Science, Berlin, Germany \\ Correspondence to: Franz P. Hessel. Ernst-Reuter-Platz 10, D-10587 Berlin, Germany. Email: franz.hessel@srh.de.
}

\begin{abstract}
Heart failure (HF) is a frequent cause of morbidity and mortality worldwide. The prevalence of HF increases, and in high-income countries, $1-2 \%$ of total healthcare expenditure is spent on HF. This article gives an overview on the impact of $\mathrm{HF}$ on health-related quality of life (HRQoL) and the economic burden of HF. Those suffering from HF are associated with a substantial decrease of HRQoL compared to individuals with most other chronic diseases and to individuals without HF. Therapeutic approaches, which decrease risk factors and lead to an improvement of the clinical status of patients, have a positive effect on HRQoL of the patients. Hospitalization rates have been shown to be correlated with disease severity, mortality, and HRQoL. Inpatient treatments of HF patients are cost intensive and the most important component for the economic burden of HF, responsible for at least half of direct cost. Prevention strategies, diagnostic and therapeutic approaches should focus on avoiding need for hospitalizations, and in particular, readmissions. Outpatient care including medication represents the second largest cost component. The cost of HF varies from less than 1,000 USD per patient in low-income countries to between 5,000 and 15,000 EUR in Europe, and between 17,000 and 30,000 USD in the US. There is a lack of study results on indirect costs. All study results on the socio-economic burden of HF clearly underscore the public health relevance of HF, showing a large economic burden for healthcare systems all over the world and a considerable impact on patients' HRQoL. The results on HRQoL are relatively homogeneous, but there are large differences across countries in respect of the economic burden they have to bear. Despite the large number of studies on the socio-economic consequences of HF further research is necessary, especially on indirect cost and for lowand middle-income countries. Future studies would benefit from a greater standardization of methods and presentation of results.
\end{abstract}

Keywords: Heart failure (HF); health-related quality of life (HRQoL); cost of illness; socio-economic burden

Submitted Feb 25, 2020. Accepted for publication Aug 20, 2020.

doi: $10.21037 / \mathrm{cdt}-20-291$

View this article at: http://dx.doi.org/10.21037/cdt-20-291

\section{Introduction}

The socio-economic impact of a disease is a structured way of showing the consequences of a specific health state or a disease for society as a whole and for various parties such as patients, healthcare providers and healthcare payers. This article aims to give an overview on the socioeconomic burden of HF. The scientific evidence on epidemiological measures such as prevalence, incidence and mortality, and humanistic parameters such as healthrelated quality of life (HRQoL) and the economic burden of $\mathrm{HF}$ is summarized.

\section{Epidemiology of cardiovascular disease (CVD) and heart failure (HF)}

The term CVD summarizes HF, ischemic heart disease, stroke, peripheral heart disease and a number of other cardiac and vascular conditions (1). CVD is the leading cause of mortality and morbidity. In 2017, CVD was estimated to be responsible for 17.8 million deaths worldwide (1), what stands for nearly one third of the global mortality. The latest issue of the annual statistics summarized by the American Heart Association reports that about $48 \%$ of the adults in the U.S. in 2016 had CVD (2). 


\section{Incidence and prevalence of $\mathrm{HF}$}

$\mathrm{HF}$ is defined as a clinical syndrome characterized by a reduced ability of the heart to pump or fill with blood, manifested as increased left ventricular filling pressure. HF is classified into three subtypes, namely HF with reduced ejection fraction (HFrEF), HF with preserved ejection fraction $(\mathrm{HFpEF})$ and $\mathrm{HF}$ mid-range ejection fraction (HFmrEF) $(3,4)$. Although this classification is based on clinical parameters it is important for socio-economic aspects of $\mathrm{HF}$ as well.

HF patients represent about $5 \%$ of the CVD patients. HF globally affects approximately 23 million people including 6 million people in the USA and more than 15 million people in Europe (4). The prevalence of HF is between $1-2 \%$ of the adult population in industrialized high-income countries, rising to $10 \%$ in a population over 70 years of age. At the age of 55 years the risk to develop HF over the remaining lifetime it is estimated to be $33 \%$ for men and $28 \%$ for women (3).

The prevalence and incidence of $\mathrm{HF}$ is influenced by a number of risk factors such as age, hypertension, chronic heart disease, obesity, hyperlipidemia, and diabetes (3). The prevalence has increased over the past decades. Associated with the increasing proportion of elderly people and the improvements in diagnosis and therapy, it is expected that there will be a further rise (5). Incidence is relatively stable over time, with numbers of 500,000 to 600,000 newly diagnosed cases per year. This indicates that the management of risk factors by focused primary prevention strategies was successful in delaying the development of $\mathrm{HF}$ (5).

Most of the studies on prevalence and incidence of HF focused on the US and European population. A recent worldwide population-based study found relevant differences in long-term outcomes of HF patients differentiated by region, sex, place of diagnosis, and socioeconomic status. The authors concluded that tailored management strategies could significantly improve the long-term outcome of HF patients (6).

\section{Mortality}

There is no doubt that HF significantly lowers overall survival probability. A large meta-analysis estimated a $40.2 \%$ mortality of HF patients during a median follow-up of 2.5 years (7). Data from other population-based studies such as the Framingham Heart Study and the Olmsted Study confirmed the high mortality of $\mathrm{HF}$ with rates of $50-59 \%$ in men and 30-46\% in women in the 1990s (8-10). Analysis of large administrative datasets from a US Medicare population and a UK NHS population demonstrated only moderate improvements in survival from 1994 to 2003 in the US and from 2000 to 2017 in the UK $(11,12)$.

\section{Regional variation of mortality}

HF-specific mortality varies considerably across regions and countries. Based on the recently published multinational cohort study INTER-CHF, the overall 1-year mortality is $16.5-7 \%$ in China, 9\% in South America and the Middle East, 34\% in African countries (13). The authors concluded that differences in health-care infrastructure, quality and access, or environmental and genetic factors might be responsible for the regional differences in HF mortality (13).

A comparable study on Asian countries confirmed the regional differences in mortality, associated with the economic differences across the countries. In the subsample of patients with HFrEF, Southeast Asia has the highest crude all cause 1-year mortality (13.6\%), compared with $8.9 \%$ in Northeast Asia and $8.3 \%$ in South Asia. Individual countries with the highest mortality were Indonesia (21.4\%) and the Philippines (14.3\%), despite relatively young populations. On the other hand, Japan, with a relatively elderly population, had the lowest 1 - year mortality rate of $4.4 \%$ (14).

Generally, the vast majority of studies analyzing the epidemiology of HF have been performed in western industrialized high-income country populations. The lack of scientific evidence on epidemiology of HF for other regions might be caused by the lower priority for financing institutions as well as the availability of administrative data. Nevertheless, the high mortality in African countries and India underscore the need for further studies and tailored disease management in these regions (6).

\section{Humanistic burden of HF}

The term "humanistic burden" summarizes the impact of an illness on patients' HRQoL, the activities of daily living, caregiver health and caregiver quality of life. It also takes into account patients' treatment satisfaction and compliance with their specific treatment regimen. More than $90 \%$ of publications on the humanistic burden of HF focused on HRQoL. Outcome measures perceived and reported directly by the patients influence the therapy decision making process. These assumptions are supported by 
empirical evidence, e.g., the SHAPE study showed that two thirds of the normal population prefer improved HRQoL to length of survival (15). Previous US studies on HF patients confirmed this preference $(16,17)$ and underscored the importance of HRQoL as an outcome parameter.

\section{HRQoL}

On the individual patient level, HRQoL includes the physical and mental health over time. HRQoL is put into effect by disease-specific and generic measures which are perceived and usually reported by the patients and then evaluated using validated questionnaires. Scientific evidence clearly shows that those with HF are associated with a substantial decrease of HRQoL compared to individuals with most other chronic diseases and to individuals without HF (18-20). A number of studies showed that more severe NYHA stages are associated with a lower HRQoL. The results were relatively consistent across the different approaches to measure HRQoL such as generic instruments (e.g., EQ-5D, SF-12, SF-36) or disease-specific instruments (e.g., MLHFQ, KCCQ) (21-24). The impact of the ejection fraction on generic HRQoL was demonstrated in one study using SF-26 (25). Another study with the disease-specific Kansas City Cardiomyopathy Questionnaire (KCCQ) found no significant difference between HF patients with an ejection fraction lower than 40 or higher than 50 (23). Specific clinical consequences of HF such as poor mental health, comorbidities, as well as the need for hospitalization, have been demonstrated to be associated with worse HRQoL in patients with HF (20).

Therapeutic approaches which improve the clinical status of patients with HF also have a positive effect on HRQoL of the patients. A large number of studies can be found on the HRQoL consequences of specific pharmaceutical interventions. A recent review from Giles et al. gives a comprehensive overview (20). This relatively extensive body of scientific evidence from this large number of studies may be a consequence of the acknowledgement of HRQoL as patient-relevant outcome measure for pricing and reimbursement decision-making processes for pharmaceuticals in many countries. Practically for all new pharmaceuticals, HRQoL studies are included in the standard program of clinical development of the companies. For example, the uses of angiotensin II receptor blocker, angiotensin converting enzyme inhibitor, or beta blockers in patients with HF were shown to be positively correlated with HRQoL (26-29).
The use of left ventricular assist devices as bridge to transplantation is a typical example for a positive effect of the use of a medical device in HRQoL of patients with advanced HF. As summarized in a systematic review, left ventricular assist devices significantly improved quantity of life as well as the HRQoL for patients with HF. Improvements in HRQoL are seen 3 months after implantation and remain throughout the duration of support (30).

Several studies investigated the suitability of HRQoL as a predictive factor of mortality and long-term outcomes in patients with HF. Data from European countries and the USA demonstrated an association of HRQoL scores and mortality and survival without cardiac events (31-35).

\section{Hospitalization rate}

In most healthcare systems statistics on hospitalization are easily available. The hospitalization rates for patients with HF have been shown to be correlated with disease severity, mortality, HRQoL, as well as long-term treatment costs. Most data are available for 1-year hospitalization rates of HF patients, either for HF treatment or all-cause hospitalization. Depending on a number of factorsage, severity of HF, previous hospitalization, country and therapeutic strategy, HF-related hospitalization occurs in up to $25 \%$ of the HF patients, whereas all-cause hospitalizations were observed in up to $60 \%$ (20). Strong predictors for hospitalization seem to be a previous hospital admission and comorbidities. In the majority of the cases $\mathrm{HF}$ is not the main diagnosis of admission. Frequent first diagnoses of admission were pulmonary disease, renal failure, and infections $(5,36)$.

Furthermore, between $15 \%$ and $30 \%$ of patients hospitalized for HF are likely to be readmitted within a month after discharge from hospital. The rates for HF-related readmission ranged from $13.8-46.1 \%$ depending on study population and country-specific healthcare setting (20).

\section{Economic burden of HF}

Health economic studies are differentiated according to the aim of the study and the target group for the study results. To compare two or more defined healthcare technologies or healthcare strategies, economic evaluation studies or cost-effectiveness simulation models became a standard requirement or at least a helpful add-on for pricing and reimbursement decisions in many countries. The results of 
health economic evaluation studies are cost-outcome-ratios such as incremental cost per life-year gained per patient or population treated, or incremental cost per quality-adjusted life-year (QALY). Use of these parameters is an attempt to express the value of a defined healthcare measure.

The estimation of the economic burden of a disease follows a different methodological concept. Instead of calculation of the additional cost to gain a unit of medical outcome, only the costs associated with a specific disease or a specific health status are calculated. The average economic consequences (expenses and savings) of a disease or in a defined health state are calculated. Outcomes are indirectly considered as changes in outcome might lead to additional costs or savings. The costs can be expressed as costs per average patient or cost for a whole population.

The calculation of the costs can be limited to the most relevant cost component, which in case of HF is hospitalization. Further components of the direct cost of HF are outpatient care, medication, rehabilitation, professional nursing care and informal care. Indirect costs are reflecting the productivity loss expressed as 'presenteeism' (i.e., working while sick), sick leave, early retirement, and premature mortality. The total cost of illness of a disease is estimated by the total amount of direct and indirect costs associated with the disease.

\section{Cost of inpatient care}

Inpatient hospital care is the major cost driver in HF. In multiple studies across different countries and regions, it was consistently reported to be the largest contributor to direct costs. Variations of systems, economies, populations as well as statistical and health economic methods, lead to a wide variation of results across studies.

Even after adjustment for currency and the year of calculation, the absolute cost for hospitalization varies so widely across the studies that a direct comparison seems to be inappropriate (37). The lowest cost for hospitalization due to HF of about 900 USD per year was reported for South Korea (38), the highest number of about 125,000 USD per patient per year was calculated for the US healthcare system (39). According to a recent review in-patient costs for HF patients in European countries range from about 5,000 USD to about 18,000 USD (37).

The percentage of hospitalization cost from total costs varies between $47 \%$ and $92 \%$, after adjustment for currency and year (20,37). But even keeping in mind these wide variations, the available scientific evidence leaves no room for doubt that hospital treatment and hospitalizations in HF are cost-intensive and the most important component for the economic burden of HF.

\section{Cost for outpatient care}

Practically all comprehensive health economic studies on HF presented data on the cost of outpatient care and medications (37). Other components of the direct costs such as the cost for nursing care, rehabilitation or specific lab tests have rarely been in the focus of health economic studies on HF. In most studies it remains unclear whether the cost of outpatient care and medication is limited to HFrelated care. This methodological lack of clarity complicates direct comparisons of the study results.

The reliability of direct comparisons of absolute cost numbers for various countries is limited. The relative proportion of main cost components to total costs seems to be easier to transfer from one country to another and more appropriate to compare. Derived from the results of a comprehensive and concise US study (40), a typical sample of different cost components was calculated as follows: hospitalizations $47 \%$; medications $20 \%$; office-based care $14 \%$; home nursing care $8 \%$, outpatient care $5 \%$, other $8 \%$.

Comprehensive and valid numbers of samples of cost components for other countries are not available.

\section{Indirect/societal cost of $\mathrm{HF}$}

The scientific body of evidence on indirect cost of $\mathrm{HF}$ is limited to three publications (41-43). An analysis of administrative data for a Medicare population from the US (41) reported total annual indirect cost of HF of 10.2 billion USD respectively about 1,860 USD per patient for the year 2012. Only one study could be identified reporting all relevant components of indirect costs. Total indirect costs of HF in Poland were 945 million EUR in 2015. The main driver of indirect costs of HF was premature mortality (about 60\%) followed by 'presenteeism' $(22 \%)$, disability $(12 \%)$ and sick leave $(4.0 \%)$. The cost of caregivers' absenteeism was below $0,01 \%$ of the indirect costs and therefore considered to be negligible (42). In contrast to the results from Poland, a Spanish study underscored the relevance of family caregivers' costs by being responsible for about two third of total HF cost (43).

\section{Cost of illness}

Three recent systematic reviews summarize the current 
scientific evidence on the total cost of HF $(20,37,44)$. The number of studies included in the reviews varied between 16 and 35. All three reviews remarked that there was a broad range of the annual costs per HF patient. The lowest numbers of all studies with less than 1,000 USD are presented for Nigeria and South Korea $(38,45)$. The annual cost per patient in Europe varies from about 5,000 EUR to 15,000 EUR (37) without obvious reasons for such large discrepancies. The annual direct cost per HF patient in the US tends to be the highest in the world. The results of the studies range from 17,000 USD to 30,000 USD $(20,37)$.

Several publications refer to $1-2 \%$ of the overall healthcare budget spent for HF (37). Only for a few countries are valid study-results on the country-specific economic burden of HF available. There is almost a complete lack of data regarding middle and low-income countries, although they represent over $80 \%$ of the world's population. For most countries in the world epidemiological and economic statistics are missing or not sufficient to estimate reliable cost of illness measures. The majority of direct cost of HF is paid out-of-pocket and therefore not regularly documented. Due to a lack of all-encompassing reporting systems for sick leave and retirement it is impossible to estimate indirect cost by using administrative data. Only a single rough estimate could be identified which used a prognostic simulation model (46). The global annual cost of HF for the year 2012 was estimated to be $\$ 108$ billion. Direct costs were estimated to be $\$ 65$ billion, indirect costs $\$ 43$ billon; $86 \%$ of the global HF cost is spent in high income countries. The authors assume a large difference of the ratio of direct and indirect cost (46).

Rapid economic development and changes of morbidity patterns in high-population countries, especially China and Brazil, might have changed this picture over the last decade. High increase of healthcare spending per capita of up to $10 \%$ per year is likely to affect the cost of $\mathrm{HF}$ and its distribution pattern as well (47).

\section{Approaches to the management of HF costs}

In coronary heart disease primary prevention with medications such as statins, as well as non-pharmaceutical strategies such as smoking cessation, has been a popular topic of health economic studies for many decades. Most HF efforts are targeted at treatment and secondary prevention (48). Again, most studies have been performed for western high-income countries, mainly for pharmaceutical approaches; also for low and middle- income countries a considerable number of studies have demonstrated the feasibility and cost-effectiveness of programs aiming for example at salt intake and tobacco consumption (49). In the long run effective prevention strategies often result in overall savings due to the number of cases and complications avoided.

Cost-effective diagnostic approaches in HF aim at different targets. One target might be to attain a valid 'ruling-out' procedure in emergency situations. A fast and valid differentiation between positive HF patients and those with other underlying diseases leads to a decrease of hospital cost. A second target - more relevant from a public health perspective-aims at the management of HF patients, resulting in a decrease of hospital admissions and the use of further healthcare resources by focused, biomarker-guided treatment. A third and future target is the screening of individuals with elevated risk for HF to achieve early detection and treatment, and for the avoidance of unnecessary treatment of healthy individuals or those who are falsely classified as having HF.

Most health economic evaluation studies on HF patients focus on therapy. All HF patients should receive recommended evidence-based therapies suitable for, and according, to the preferences of the individual patient. Cost-effectiveness studies always compare alternative strategies. They cannot replace the individual clinical decision, but they can add information important for pricing and reimbursement decisions for pharmaceuticals and devices to avoid unjustified prices caused by the lack of competition between manufacturers.

\section{Discussion and outlook}

Published guidelines on HF summarize evidencebased strategies for prevention, diagnosis, therapy and rehabilitation. Health economic evaluation studies do not contradict the evidence derived from clinical or patientreported outcome studies, but add the economic dimension expressed in additional cost or saving due to the use of a defined medication or medical device.

This review summarizes the current picture of the socioeconomic burden of HF focusing on the consequences on the HRQoL, hospitalizations, and the expenses for medical care of patients with HF. This paper is meant to give a narrative overview of the literature and the study results on a number of public health aspects of HF. A meta-analysis of the published study results would reach a higher level of evidence but requires a broader database and a much more complex methodological approach. 
Medical decision-making processes affect individual patients. They should be based on clinical evidence and patient preferences, rather than made on the basis of individual or local economic interests. But it seems to be unavoidable to take into consideration the scarcity of monetary resources as well as the quality of life of patients and professionals for the overall public health goals, the legal framework, pricing and reimbursement decisions.

Hundreds of scientific studies on socio-economic consequences of HF have been conducted and published. This number alone is underscoring the importance of the disease. Not only the number but also the results of the studies clearly highlight the importance of HF, showing a large economic burden of HF for healthcare systems all over the world and a considerable impact on HRQL. Not surprisingly the results on HRQoL are relatively homogeneous, but there is a huge difference across countries in the absolute economic burden, which is not only explained by the differences in gross domestic product (GDP) estimations, but also due to the differences in the percentage of GDP spent for healthcare. Especially in low and middle-income countries the majority of healthcare cost must be covered out-of-pocket of patients and families. There is a lack of scientific evidence with regards to this aspect. In general, the number of cost of illness and HRQoL studies for low and middle income countries is very low. For the vast majority of these countries there are no studies at all. As a lot of studies are supported by industry grants, the priority is put on the large markets. In response to this development, global non-profit institutions such as World Health Organization or Bill and Melinda Foundation started large global study initiatives (47). Also, the implementation of universal healthcare programs, e.g., in the Philippines or Indonesia, will improve the availability of administrative data and enable analyses, also on HF.

The results of the identified studies show such a broad range. Only a small percentage of the variations are due to the differences across healthcare systems and the lack of data. The main cause is seen in the heterogeneity of the study methods. Future studies on the socio-economic burden of HF should use a standardized methodological approach regarding selection criteria of HF patients and data, the inclusion of different cost components, the statistical approach and the presentation of results $(20,37,44)$.

\section{Conclusions}

All preventive, diagnostic, therapeutic and rehabilitation approaches for HF which demonstrated a sufficiently evidence-based outcome-gain should be part of medical care. Nevertheless, the allocation of scarce monetary and human resources should be made with an awareness of the socio-economic consequences.

The body of scientific evidence on the humanistic burden of HF by HRQoL and the economic burden expressed in estimations of the cost of hospitalization or the cost of illness due to HF clearly shows a considerable and continuously growing socio-economic burden of HF. This trend for rising costs has been observed for high-income countries over the last 20 years and future demographic developments predict further increases in the future. It is assumed that low and middle-income countries will follow

Inpatient treatment and hospitalization are the main driver for a decrease of HRQoL and represent the largest cost component in industrialized countries in HF. Prevention strategies, diagnostic and therapeutic approaches including hospital care should consequently focus on avoiding the need for hospitalizations, and in particular, readmissions.

Further research on indirect costs and costs of nursing care as well as studies in low- and middle-income countries are recommended. Future studies would benefit from a greater standardization of methods and presentation of HRQoL and cost of illness results.

\section{Acknowledgments}

Funding: None.

\section{Footnote}

Provenance and Peer Review: This article was commissioned by the Guest Editor (Roland Hetzer) for the series "Heart Failure in the Young and Old: Insights into Various Therapies" published in Cardiovascular Diagnosis and Therapy. The article has undergone external peer review.

Conflicts of Interest: The author has completed the ICMJE uniform disclosure form (available at http://dx.doi. org/10.21037/cdt-20-291). The series "Heart Failure in the Young and Old: Insights into Various Therapies" was commissioned by the editorial office without any funding or sponsorship. The author has no other conflicts of interest to declare.

Ethical Statement: The author is accountable for all aspects of the work in ensuring that questions related 
to the accuracy or integrity of any part of the work are appropriately investigated and resolved.

Open Access Statement: This is an Open Access article distributed in accordance with the Creative Commons Attribution-NonCommercial-NoDerivs 4.0 International License (CC BY-NC-ND 4.0), which permits the noncommercial replication and distribution of the article with the strict proviso that no changes or edits are made and the original work is properly cited (including links to both the formal publication through the relevant DOI and the license). See: https://creativecommons.org/licenses/by-nc-nd/4.0/.

\section{References}

1. GBD 2017 DALYs and HALE Collaborators. Global, regional, and national disability-adjusted life-years (DALYs) for 359 diseases and injuries and healthy life expectancy (HALE) for 195 countries and territories, 1990-2017: a systematic analysis for the Global Burden of Disease Study 2017. Lancet 2018;392:1859-922.

2. Benjamin EJ, Muntner P, Alonso A, et al. Heart Disease and Stroke Statistics-2019 Update: A Report From the American Heart Association. Circulation 2019;139:e56-528.

3. Ponikowski P, Voors AA, Anker SD, et al. 2016 ESC Guidelines for the diagnosis and treatment of acute and chronic heart failure: The Task Force for the diagnosis and treatment of acute and chronic heart failure of the European Society of Cardiology (ESC). Eur J Heart Fail 2016;18:891-975.

4. Savarese G, Lund LH. Global Public Health Burden of Heart Failure. Cardiac Failure Review 2017;3:7-11.

5. Roger VL. Epidemiology of heart failure. Circ Res 2013;113:646-59.

6. Lawson CA, Zaccardi F, Squire I, et al. 20-year Trends in Cause-Specific Heart Failure Outcomes by Sex, Socioeconomic Status, and Place of Diagnosis: A Population-Based Study. Lancet Public Health 2019;4:e406-20.

7. Pocock SJ, Ariti CA, McMurray JJ, et al. Predicting survival in heart failure: a risk score based on 39372 patients from 30 studies. Eur Heart J 2013;34:1404-13.

8. MacIntyre K, Capewell S, Stewart S, et al. Evidence of improving prognosis in heart failure: Trends in case fatality in 66547 patients hospitalized between 1986 and 1995 . Circulation 2000;102:1126-31.

9. Levy D, Kenchaiah S, Larson MG, et al. Long-term trends in the incidence of and survival with heart failure. N Engl
J Med 2002;347:1397-402.

10. Roger VL, Weston SA, Redfield MM, et al. Trends in heart failure incidence and survival in a community-based population. JAMA 2004;292:344-50.

11. Curtis LH, Whellan DJ, Hammill BG, et al. Incidence and Prevalence of Heart Failure in Elderly Persons, 1994-2003 Arch Intern Med 2008;168:418-24.

12. Taylor CJ, Ordóñez-Mena JM, Roalfe AK, et al. Trends in survival after a diagnosis of heart failure in the United Kingdom 2000-2017: population based cohort study. BMJ 2019;364:1223.

13. Dokainish H, Teo K, Zhu J, et al. Global mortality variations in patients with heart failure: results from the International Congestive Heart Failure (INTER$\mathrm{CHF}$ ) prospective cohort study. Lancet Glob Health 2017;5:e665-72.

14. MacDonald MR, Tay WT, Teng TK, et al. Regional Variation of Mortality in Heart Failure With Reduced and Preserved Ejection Fraction Across Asia: Outcomes in the ASIAN-HF Registry. J Am Heart Assoc 2020;9:e012199.

15. Remme WJ, McMurray JV, Rauch B, et al. Public awareness of heart failure in Europe: first results from SHAPE. Eur Heart J 2005;26:2413-21.

16. Lewis EF, Johnson PA, Johnson W, et al. Preferences for quality of life or survival expressed by patients with heart failure. J Heart Lung Transplant 2001;20:1016-24.

17. MacIver J, Rao V, Delgado D, et al. Choices: a study of preferences for end-of-life treatments in patients with advanced heart failure. J Heart Lung Transplant 2008;27:1002-7.

18. Lesman-Leegte I, Jaarsma T, Coyne JC, et al. Quality of life and depressive symptoms in the elderly: a comparison between patients with heart failure and ageand gender-matched community controls. J Card Fail 2009;15:17-23.

19. Peters M, Crocker H, Dummett S, et al. Change in health status in long-term conditions over a one year period: a cohort survey using patient-reported outcome measures. Health Qual Life Outcomes 2014;12:123.

20. Giles L, Freeman C, Field P, et al. Humanistic burden and economic impact of heart failure - a systematic review of the literature. F1000Research 2019;8:859.

21. Franzén K, Saveman BI, Blomqvist K. Predictors for health related quality of life in persons 65 years or older with chronic heart failure. Eur J Cardiovasc Nurs 2007;6:112-20.

22. Spertus JA, Jones PG, Kim J, et al. Validity, reliability, and responsiveness of the Kansas City Cardiomyopathy 
Questionnaire in anemic heart failure patients. Qual Life

Res 2008;17:291-8.

23. Joseph SM, Novak E, Arnold SV, et al. Comparable performance of the Kansas City Cardiomyopathy Questionnaire in patients with heart failure with preserved and reduced ejection fraction. Circ Heart Fail 2013;6:1139-46.

24. Kularatna S, Byrnes J, Chan YK, et al. Comparison of contemporaneous responses for EQ-5D-3L and Minnesota Living with Heart Failure; a case for disease specific multiattribute utility instrument in cardiovascular conditions. Int J Cardiol 2017;227:172-6.

25. Edelmann F, Stahrenberg R, Gelbrich G, et al. Contribution of comorbidities to functional impairment is higher in heart failure with preserved than with reduced ejection fraction. Clin Res Cardiol 2011;100:755-64.

26. Wolfel EE. Effects of ACE inhibitor therapy on quality of life in patients with heart failure. Pharmacotherapy 1998;18:1323-34.

27. Linde C, Leclercq C, Rex S, et al. Long-term benefits of biventricular pacing in congestive heart failure: Results from the MUltisite STimulation in cardiomyopathy (MUSTIC) study. J Am Coll Cardiol 2002;40:111-8.

28. Curiati JA, Bocchi E, Freire JO, et al. Meditation reduces sympathetic activation and improves the quality of life in elderly patients with optimally treated heart failure: A prospective randomized study. J Altern Complement Med 2005; 11:465-72.

29. Davies EJ, Moxham T, Rees K, et al. Exercise based rehabilitation for heart failure. Cochrane Database Syst Rev 2010;(4):CD003331.

30. Maciver J, Ross HJ. Quality of life and left ventricular assist device support. Circulation 2012;126:866-74.

31. Kosiborod M, Soto GE, Jones PG, et al. Identifying heart failure patients at high risk for near-term cardiovascular events with serial health status assessments. Circulation 2007;115:1975-81.

32. Hoekstra T, Jaarsma T, van Veldhuisen DJ, et al. Quality of life and survival in patients with heart failure. Eur J Heart Fail 2013;15:94-102.

33. Chamberlain AM, McNallan SM, Dunlay SM, et al. Physical health status measures predict all-cause mortality in patients with heart failure. Circ Heart Fail 2013;6:669-75.

34. Di Giulio P, Network of Nurses of GISSI-HF. Should patients' perception of health status be integrated in the prognostic assessment of heart failure patients? A prospective study. Qual Life Res 2014;23:49-56.

35. Wu JR, Lennie TA, Frazier SK, et al. Health-related quality of life, functional status, and cardiac event-free survival in patients with heart failure. J Cardiovasc Nurs 2016;31:236-44.

36. Blecker S, Paul M, Taksler G, et al. Heart failure associated hospitalizations in the United States. J Am Coll Cardiol 2013;61:1259-67.

37. Lesyuk W, Kriza C, Kolominsky-Rabas P. Cost-of-illness studies in heart failure: a systematic review 2004-2016. BMC Cardiovascular Disorders 2018;18:74.

38. Lee H, Oh SH, Cho H, et al. Prevalence and socioeconomic burden of heart failure in an aging society of South Korea. BMC Cardiovasc Disord 2016;16:215.

39. Dunlay SM, Shah ND, Shi Q, et al. Lifetime costs of medical care after heart failure diagnosis. Circ Cardiovasc Qual Outcomes 2011;4:68-75.

40. Echouffo-Tcheugui JB, Bishu KG, Fonarow GC, et al. Trends in health care expenditure among US adults with heart failure: The Medical Expenditure Panel Survey 2002-2011. Am Heart J 2017;186:63-72.

41. Voigt J, Sasha John M, Taylor A, et al. A reevaluation of the costs of heart failure and its implications for allocation of health resources in the United States. Clin Cardiol 2014;37:312-21.

42. Łyszczarz B. Indirect costs and public finance consequences of heart failure in Poland, 2012-2015. BMC Public Health 2018;18:1130.

43. Delgado JF, Oliva J, Llano M, et al. Health care and nonhealth care costs in the treatment of patients with symptomatic chronic heart failure in Spain. Rev Esp Cardiol 2014;67:643-50.

44. Shafie AA, Tan YP, Ng CH. Systematic review of economic burden of heart failure. Heart Fail Rev 2018;23:131-45.

45. Ogah OS, Stewart S, Onwujekwe OE, et al. Economic burden of heart failure: investigating outpatient and inpatient costs in Abeokuta, Southwest Nigeria. PLoS One 2014;9:e113032.

46. Cook C, Cole G, Asaria P, et al. The annual global economic burden of heart failure. Int $\mathrm{J}$ Cardiol 2014;171:368-76.

47. Global Burden of Disease Health Financing Collaborator Network. Past, present, and future of global health financing: a review of development assistance, government, out-of-pocket, and other private spending on health for 195 countries, 1995-2050. Lancet 2019;393:2233-60.

48. Butler J. Primary Prevention of Heart Failure. ISRN Cardiol 2012;2012:982417. 
49. Aminde LN, Takah NF, Zapata-Diomedi B, et al. Primary and secondary prevention interventions for cardiovascular disease in low-income and middle-income countries:

Cite this article as: Hessel FP. Overview of the socioeconomic consequences of heart failure. Cardiovasc Diagn Ther 2021;11(1):254-262. doi: 10.21037/cdt-20-291 a systematic review of economic evaluations. Cost Eff

Resour Alloc 2018;16:22. 